\title{
Reliability Index and Load/Resistance Factors of Reinforced Soil Structures
}

\author{
Masahiro SHINODA, Ph.D. \\ Researcher, \\ Masaru TATEYAMA, Ph.D. \\ Laboratory Head, \\ Foundation \& Geotechnical Engineering, Structures Technology Division
}

\begin{abstract}
This study is the first reported application of several reliability analyses to investigate the structural reliability of typical reinforced soil structures with variable heights in sliding and overturning modes, using classical or advanced reliability approaches. In this study, the sensitivities of backfill soil properties (unit weight and friction angle) and tensile strength of reinforcement to the reliability index were investigated by changing the averages and coefficients of variation. An allowable reliability index in accordance with the conventional safety factor was proposed with the corresponding tensile strength of reinforcement. The recommended load and resistance factors were calibrated based on the above allowable reliability index by the advanced first-order and second-moment (AFOSM) method with Monte Carlo stratified sampling simulation results. The recommended load and resistance design factors based on the reliability analysis showed a reasonably constant value independent of soil property and wall height but dependent on failure mode.
\end{abstract}

Keywords: reinforced soil structures, reliability index, load factor, resistance factor

\section{Introduction}

The conventional deterministic method using the safety factor can partially evaluate the variability of soil properties using confidence values to take into account an average and standard deviation of input soil parameters, but these results in conservative design. To overcome such a problem, reliability analysis based on statistics has received attention in recent years. One advantage of working with reliability analysis is that the variability of soil parameters can be evaluated rationally and quantitatively by using the reliability index or failure probability. Another advantage of this analysis is that reliability can also be used in an economic cost-benefit analysis that takes into account design and construction costs as well as the implications of design failure. Although the advantages of reliability analysis are now generally accepted, there are still only a limited number of applications to practical problems for embankments, retaining walls and earth slopes. Only a few researches have been conducted into the design of geosynthetic-reinforced soil (GRS) walls. This study is the first reported application of several reliability analyses to investigate the structural safety of typical GRS walls with variable heights for a specific failure mode, such as sliding or overturning modes, using classical or advanced reliability approaches according to the RTRI design manual (RTRI 2000).

\section{Objective}

The principal objective of the reliability analysis described here is to present the practical load and resistance factor of limit state design (LSD) using the twowedge method according to the RTRI design manual (RTRI 2000) in sliding and overturning modes. A feature of this analysis is an explicit reliability analysis technique to calibrate practical load and resistance design factors using the less time-consuming approximate method based on effective Monte Carlo simulation results.

\section{Method for deterministic and probabilistic analyses}

\subsection{Safety factor}

The GRS structure design method adopted for Japanese railways employs the two-wedge method (RTRI 2000) for safety factor evaluation in sliding and overturning failure modes:

$$
\begin{gathered}
F S=\frac{F_{r b}+F_{r g}}{F_{d w}+F_{d s}} \text { (Sliding) } \\
F S=\frac{M_{r w}+M_{r s}+M_{r g}}{M_{d w}+M_{d s}} \text { (Overturning) }
\end{gathered}
$$

where $F_{r b}$ is the shear resistance at the bottom of the facing; $F_{r g}$ is the horizontal resistance of geosynthetic reinforcement; $F_{d w}$ is the equivalent horizontal force of the facing weight multiplied by the seismic coefficient; $F_{d s}$ is the horizontal component of the earth pressure acting on the back face of the facing; $M_{r w}$ is the resisting moment of the weight of the facing; $M_{r s}$ is the resisting moment of the soil at the base of the slice determined by the Coulomb's failure criterion; $M_{r g}$ is the resisting moment of the geosynthetic pullout resistance force; $M_{d w}$ is the driving moment of the weight of the facing; and $M_{d s}$ is the driving moment of the soil slice weight and the horizontal force due to an earthquake.

\subsection{Reliability index}

The following performance function of the corresponding failure mode was used in this study: 


$$
Z_{i}=\frac{R}{S}-1=F S-1
$$

where $Z_{i}$ is the performance function of the corresponding failure mode $i ; R$ is the component of resistance capacity and $S$ is the component of the applied load. The reliability of structures can be evaluated using the sign of the performance function. For example, reliable or safe structures have a positive performance function and unreliable or unsafe structures have zero or a negative performance function in the corresponding failure mode.

In general, it is difficult to calculate the reliability index because of the problems involved in directly computing the multidimensional integration over the irregular domain in the probabilistic space. Therefore, approximate techniques were generally adopted to calculate the reliability index or failure probability. One efficient approximate approach is the advanced first-order and second-moment (AFOSM) method in which the Taylor series expansion of the performance function is linearized at some point on the failure surface (i.e. $X_{1}{ }^{*}, X_{2}{ }^{*}, \cdots, X_{n}{ }^{*}$ ). The linearizing point is called the design point or checking point. The detailed algorithm of this approach has been reported in the reference (i.e. Hasofer and Lind 1974). Assuming that $\mathrm{n}$ random variables $\left(X_{1}, X_{2}, \cdots, X_{n}\right)$ were normally distributed and statistically independent, the limit state surface can be expanded into a first order Taylor series about design points, then the mean and standard deviation of the performance function are given by

$$
\begin{aligned}
& \mu_{Z_{i}} \approx \sum_{j=1}^{n}\left(\left.\frac{\partial g_{i}}{\partial x_{j}}\right|_{x^{*}}\right) \cdot\left(\mu_{X_{j}}-x_{j}^{*}\right) \\
& \sigma_{Z_{i}} \approx \sqrt{\sum_{j=1}^{n}\left(\left.\frac{\partial g_{i}}{\partial x_{j}}\right|_{x^{*}}\right)^{2} \cdot \sigma x_{j}^{2}}
\end{aligned}
$$

The reliability index $\beta$ can be calculated by

$$
\beta_{i}=\frac{\mu_{Z_{i}}}{\sigma_{Z_{i}}}
$$

The above reliability index is the distance from the average of the performance function to the design point, as proposed by Hasofer and Lind (1974). In this format, the calculated reliability index was independent of the form of performance function. This indicates that exactly the same reliability index can be obtained by using any definition of performance function. From Eq. (7), reliable structures have a higher average (safety factor) and lower standard deviation (variability) of the performance function. When the performance function has high average values (safety factors) but high standard deviation (variability), however, such structures are not guaranteed to have high reliability. This quantitative evaluation of the structural reliability using the reliability index is an advantage of reliability design.

\subsection{Load and resistance factors}

Equation (2) can be transformed to LSD format as follows:

$$
\phi_{R} \cdot R>\gamma_{S} \cdot S
$$

where $\phi_{R}$ is the resistance factor and $\gamma_{S}$ is the load factor. Assuming that the load and resistance were normally distributed and statistically independent, these factors can be obtained by the following formulas:

$$
\begin{aligned}
& \phi_{R}=\frac{\mu_{R}-\alpha_{R} \cdot \beta_{a} \cdot \sigma_{R}}{R_{c}} \\
& \gamma_{S}=\frac{\mu_{S}-\alpha_{S} \cdot \beta_{a} \cdot \sigma_{S}}{S_{c}}
\end{aligned}
$$

where $\beta_{a}$ is the allowable reliability index and $R_{c}$ and $S_{c}$ are the characteristic values of load and resistance, respectively. In this study, the average values were used for these characteristic values. $\alpha_{R}$ and $\alpha_{S}$ are the sensitivity factors of load and resistance, which are given by

$$
\begin{aligned}
& \alpha_{R}=\frac{\sigma_{R}}{\sqrt{\sigma_{R}^{2}+\sigma_{S}^{2}}} \\
& \alpha_{S}=\frac{\sigma_{S}}{\sqrt{\sigma_{R}^{2}+\sigma_{S}^{2}}}
\end{aligned}
$$

It can be seen that the load and resistance factors for the LSD format can be calibrated with the allowable reliability index, the average and standard deviations of load and resistance shown in Eqs. 7 and 8. The average and standard deviations of load and resistance can be calculated by the effective Monte Carlo method as explained below.

\subsection{Average and standard deviation of load and resistance}

The average and standard deviation of the performance function can be obtained by the Monte Carlo stratified sampling method. In this technique the normal cumulative distribution is divided into equal intervals, then samples are taken from each interval thus being forced to sample the entire distribution. Multiplied by each standard deviation and added to each mean, each input random variable can be generated. This approach requires less iteration to recreate the input distributions and is therefore a more efficient sampling method than the conventional sampling scheme reported by Cameron et al. (2001).

\section{Analytical model and input parameters}

Figure 1 shows analytical models of typical GRS walls. The walls are from $3 \mathrm{~m}$ to $9 \mathrm{~m}$ high. A dead load of $10 \mathrm{kN} /$ $\mathrm{m}^{2}$ was applied on the top of the backfill. Input soil properties were statistically independent random variables and normally distributed. Table 1 shows the basic soil properties, categorized into three groups in which soil cohesion was not considered. In this study, the following three reliability analyses were conducted. 

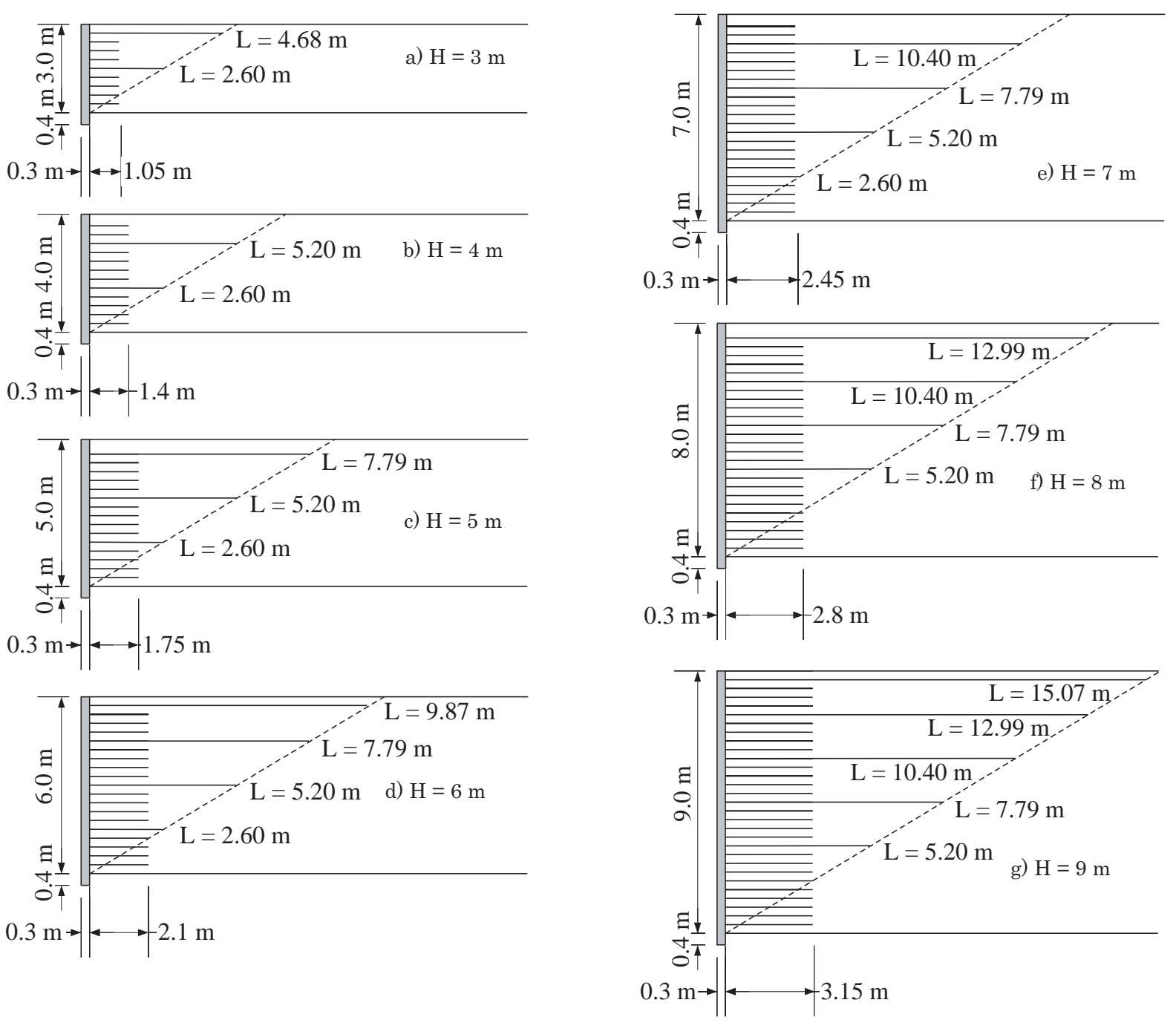

Fig. 1 Analytical models of typical GRS walls

Table 1 Classified properties of backfill soil

\begin{tabular}{c|c|c}
\hline Category & $\begin{array}{c}\text { Unit weight } \\
\left(\mathrm{kN} / \mathrm{m}^{3}\right)\end{array}$ & $\begin{array}{c}\text { Friction angle } \\
\text { (in degrees) }\end{array}$ \\
\hline Group A & 18 & 40 \\
\hline Group B & 17 & 35 \\
\hline Group C & 16 & 30 \\
\hline
\end{tabular}

\subsection{Sensitivity analysis}

The sensitivity of unit weight, friction angle and tensile strength of reinforcement on the reliability index was investigated by changing the average values and coefficients of variation, COVs, as shown in Table 2 . The unit weight and friction angle COVs were varied between 5 , 10 , and $15 \%$ for each average value. A model with a constant wall height of $7 \mathrm{~m}$ and having $\mathrm{FS}=2.0$ with the corresponding average tensile strength was adopted only for sliding mode in the sensitivity analysis.
Table 2 Input parameters for sensitivity analysis

\begin{tabular}{c|c|c|c}
\hline Item & Parameter & Average & COV \\
\hline \multirow{4}{*}{ Backfill soil } & Unit weight & $10,12.5,15$, & 0.05, \\
& $\left(\mathrm{kN} / \mathrm{m}^{3}\right)$ & $17.5,20$, & 0.1, \\
& & $22.5,25$ & 0.15 \\
\cline { 2 - 4 } & Friction angle & $20,25,30$, & 0.05, \\
& (in degree) & $35,40,45$, & 0.1, \\
& & $50,55,60$ & 0.15 \\
\hline \multirow{3}{*}{ Reinforcement } & Tensile & $10,20,30$, & 0.05, \\
& strength & $40,50,60$ & 0.1, \\
& (kN/m) & & 0.15 \\
\hline
\end{tabular}

\subsection{Allowable reliability index}

The allowable reliability index was investigated on a model with an average unit weight and friction angle shown in Table 1 , a constant COV of $10 \%$, variable wall height and FS = 2.0 with the corresponding average tensile strength in sliding and overturning modes. 


\subsection{Load and resistance factors}

The load and resistance factors of the LSD were rigorously calibrated with the above allowable reliability index in each soil group in both sliding and overturning modes. Table 1 shows the average values of unit weight and friction angle of each soil group property. The COVs of unit weight and friction angle were 5 and $10 \%$ and 5 , 10 , and $15 \%$, respectively.

\section{Results}

\subsection{Sensitivity analysis}

The reliability index corresponded to the sensitivity of unit weight and friction angle on models having a constant COV but a variable average value of input parameters with different wall heights. The sensitivity analysis results (Figs. 2a to 2c) showed that the average of the unit weight was less sensitive and friction angle was very sensitive to the reliability index. The reliability index was not influenced by the different unit weight COVs. The safety factors on the same models that were superimposed on the figures could not evaluate the soil variability appropriately even by using the same average values.

\subsection{Allowable reliability index}

Figures $3 \mathrm{a}$ and $3 \mathrm{~b}$ show the influence of soil properties and failure mode on the reliability index for models with a constant soil property $\operatorname{COV}(10 \%)$ and tensile strength of reinforcement in accordance with FS $=2.0$ for each wall height. Interestingly, the reliability index for soil properties with lower densities and friction angles was larger than those soil properties with higher densities and friction angles. A possible explanation for this trend is the relatively lower sensitivity of friction angle induced by decreasing the average, which can make the reliability index larger. The results shown in Figs. 3a and $3 \mathrm{~b}$ indicate that the reliability of structures has a minor difference even when they have the same safety factors.

\subsection{Load and resistance factors}

Load and resistance factors were calibrated by using the proposed allowable reliability index (Figs. 3a and 3b) on models having reinforcement lengths corresponding $\mathrm{FS}=1.5$ with input soil properties in each soil group (Table 1) and failure mode. Friction angle COVs of $5 \%$, $10 \%$ and $15 \%$ were used, while unit weight COVs of $5 \%$ and $10 \%$ were used because of their lower sensitivity to the reliability index. Figures $4 \mathrm{a}$ and $4 \mathrm{~b}$ show load and resistance factors in sliding mode in the soil groups of $\mathrm{A}$ and $\mathrm{C}$, respectively. Average values of the load and resistance factors show practically the same value of 1.2 and 0.6 , respectively. Load and resistance factors in overturning mode in the soil groups of $\mathrm{A}$ and $\mathrm{C}$ are plotted in Figs.

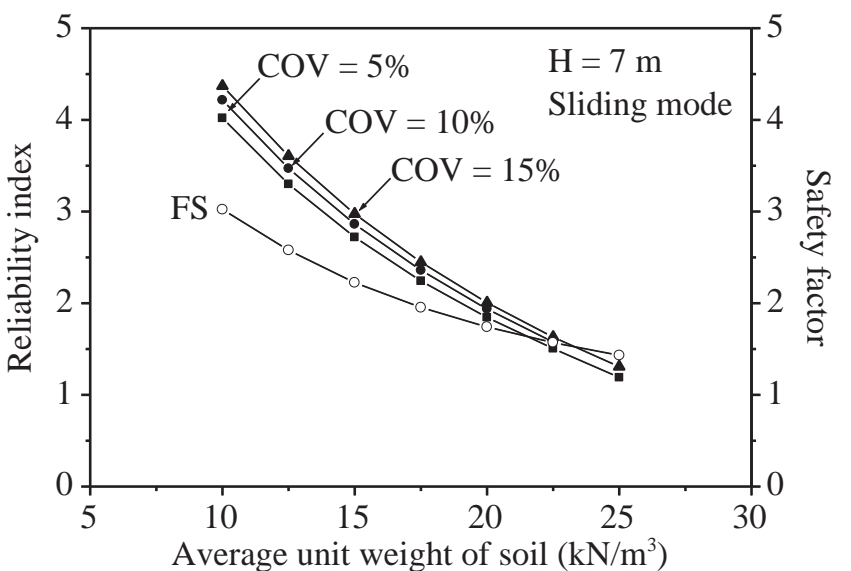

Fig. 2a Sensitivity of unit weight

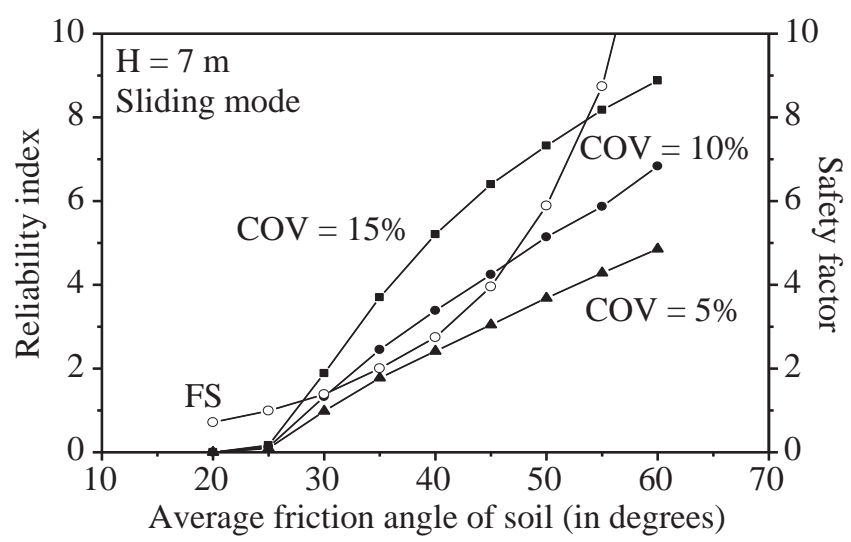

Fig. $2 b$ Sensitivity of friction angle

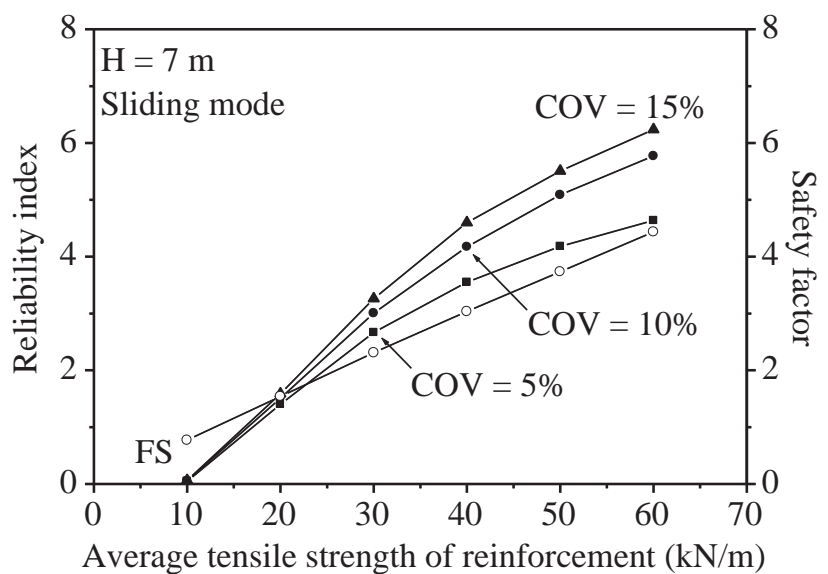

Fig. 2c Sensitivity of tensile strength of reinforcement

$4 \mathrm{c}$ and $4 \mathrm{~d}$, respectively. Average values of load and resistance factors show practically the same values of 1.4 and 0.7 , respectively. It is interesting to note that a ratio of the load factor to the resistance factor exhibited reasonably the same value of 2.0 . 


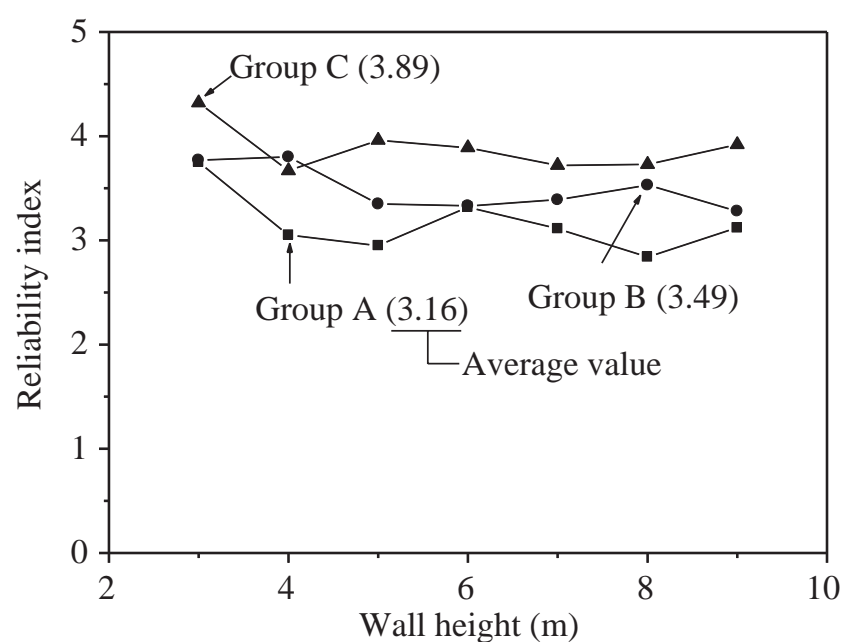

Fig. 3a Allowable reliability index in sliding mode

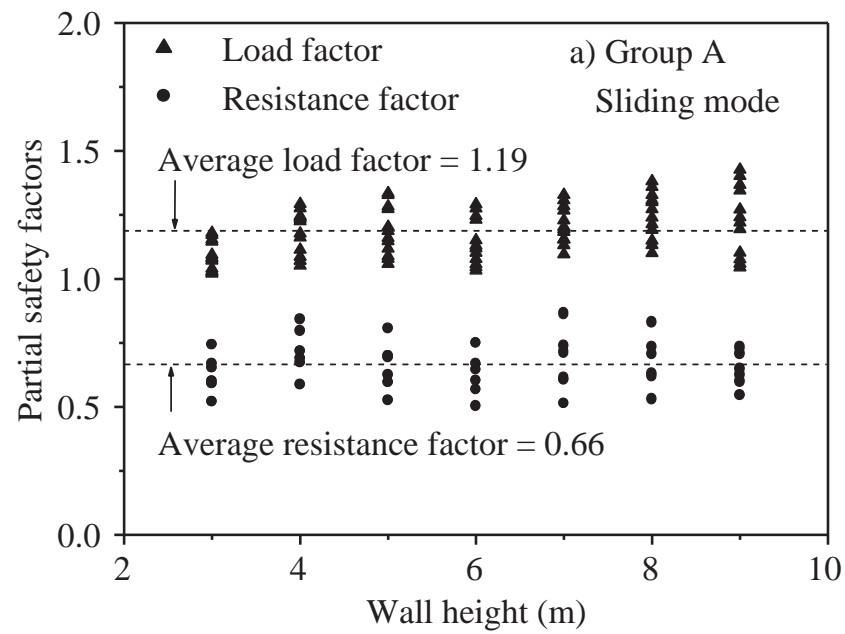

Fig. 4a Load and resistance factors in sliding and overturning modes

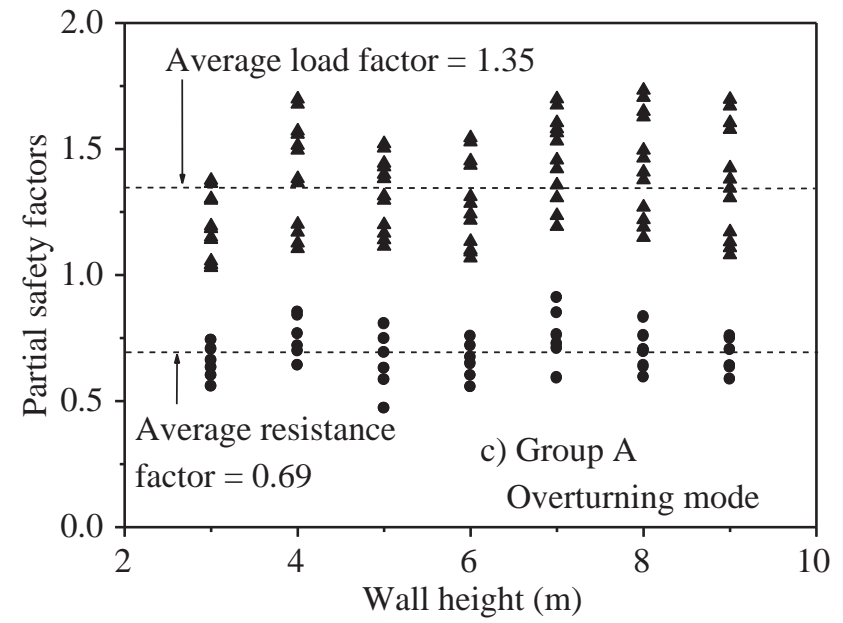

Fig. 4c Load and resistance factors in sliding and overturning modes

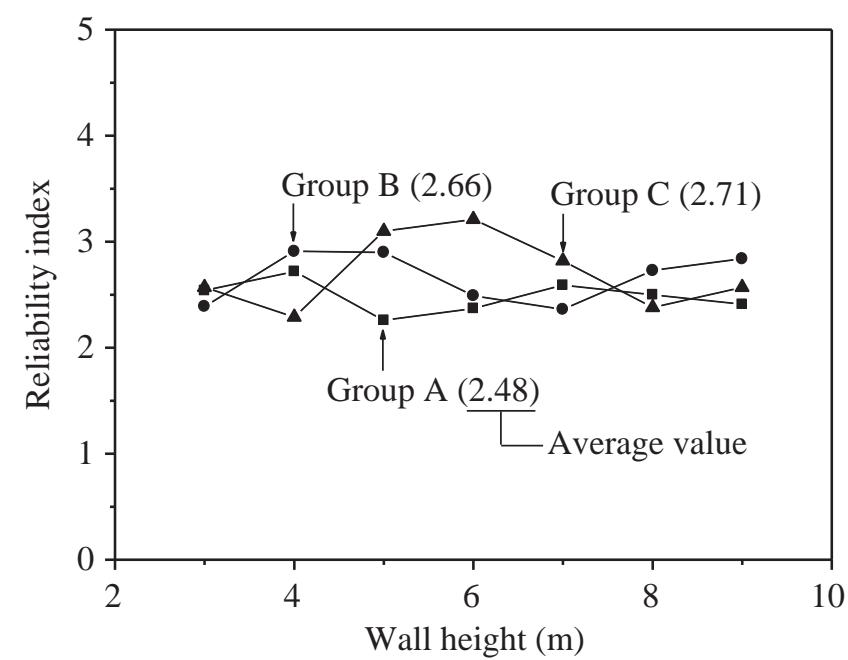

Fig. 3b Allowable reliability index in overturning mode

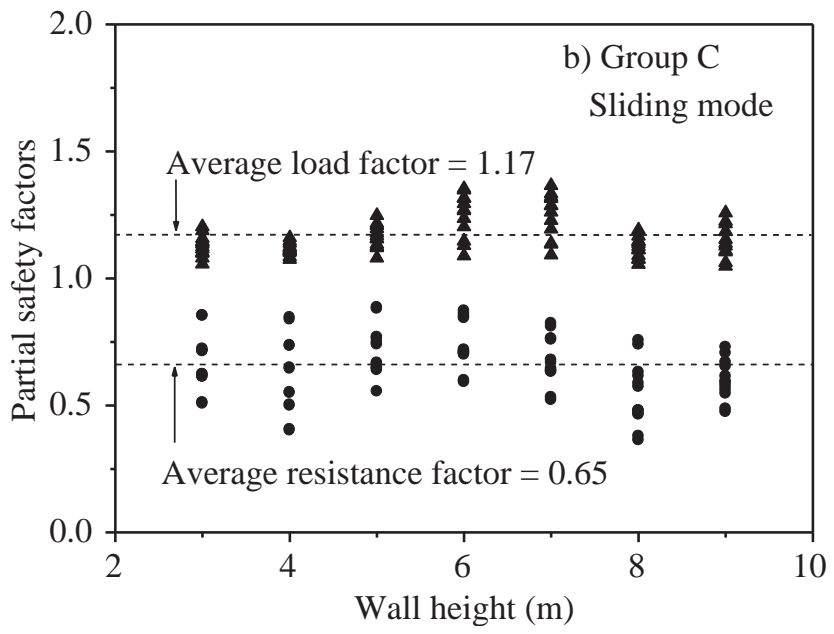

Fig. 4b Load and resistance factors in sliding and overturning modes

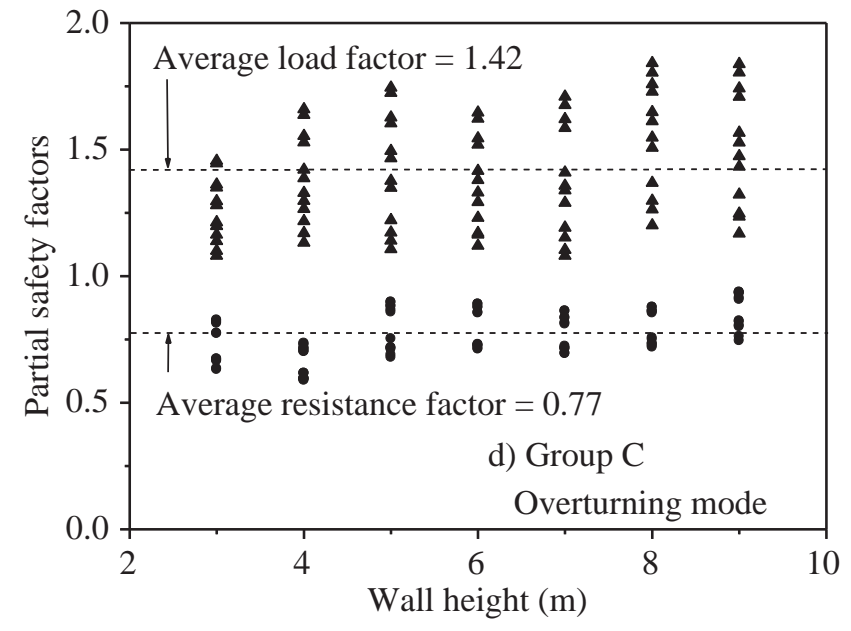

Fig. 4d Load and resistance factors in sliding and overturning modes 


\section{Conclusions}

This paper reports the results of a series of reliability analyses conducted to examine the structural reliability of geosynthetic-reinforced soil (GRS) walls in both sliding and overturning modes. The reliability index strongly depended on the average and COV of friction angle, while the unit weight COV did not affect the reliability index. The allowable reliability index showed a minor difference with soil property and failure mode with a model having the same safety factor. Based on the above results, the load and resistance factors could be calibrated reasonably. Recommended load and resistance factors in sliding mode are 1.2 and 0.6 , respectively. Recommended load and resistance factors in overturning mode are 1.4 and 0.7 , respectively. The above loud and resistance factors were independent of soil property and wall height, but dependent on failure mode.

\section{References}

1) Cameron, G., Smith, I. G. N. and Wamuziri, S. C. "An account of geotechnical risk and reliability," Proceeding of the 2001 An Earth Odyssey, pp. 1434-1440, 2001.

2) Hasofer, A. M. and Lind, N. C. "Exact and invariant second-moment code format," Journal of Engineering Mechanics Division, ASCE, Vol. 100 (1), pp.111-121, 1974.

3) RTRI. "Design Standard for Railway Earth Structures," Railway Technical Research Institute (RTRI), Maruzen, 2000, (in Japanese). 